\title{
Will a Fibrin Sealant be Effective as a Seroma Reductant and a Hemostatic Agent in Breast Reconstruction Using a Latissimus Dorsi Flap?
}

\section{Da Woon Lee, Sang Gue Kang, Min Sung Tak}

Department of Plastic and Reconstructive Surgery, Soonchunhyang Seoul Hospital, Soonchunhyang University College of Medicine, Seoul, Korea
No potential conflict of interest relevant to this article was reported.
Background The latissimus dorsi (LD) flap is widely used in breast cancer reconstruction, but donor-site morbidity is one of the major limitations of this surgery. Donor-site seroma is the most common complication. To prevent seroma formation, we consider the use of a fibrin sealant (FS) because of its hemostatic and sealing effects. In this study, we investigate the effect of a FS on seroma prevention and as a hemostatic agent at the LD donor site.

Methods A retrospective study was conducted from 2011 to 2015. Herein, we analyzed the preoperative status, changes in the hemoglobin ( $\mathrm{Hb}$ ) level according to the postoperative day, postoperative drain amount, and the drain removal time.

Results The decline in the Hb level was not statistically significantly less in the FS group than in the control group. Further, the difference in the drain amount between the 2 groups was not statistically significant either. The seroma rate and the drain removal time between the 2 groups also did not show any statistically significant difference.

Conclusions The FS does not have hemostatic effect and the drain amount reduction in the early phase of recovery and does not prevent seroma. Therefore, an empirical use of the FS alone is no longer recommended to prevent seroma. In contrast, the quilting suture has been reported to be effective in seroma prevention. Therefore, it is necessary to study the effects of a combination of quilting sutures and the FS on seroma and the other risk factors of this surgical complication.

Keywords Fibrin tissue adhesive, Seroma, Superficial back muscles

\section{INTRODUCTION}

Breast cancer is the second most common cancer in Korean women, representing $14.8 \%$ of all newly diagnosed cancers, and this proportion is gradually increasing [1]. Many flap methods are be-

Received: Mar 27, 2017 Revised: May 2, 2017 Accepted: May 3, 2017 Correspondence: Sang Gue Kang Department of Plastic and Reconstructive Surgery, Soonchunhyang Seoul Hospital, Soonchunhyang University College of Medicine, 59 Daesagwan-ro, Yongsan-gu, Seoul 04401, Korea.

E-mail: sgkang@schmc.ac.kr

Copyright $\odot 2017$ The Korean Society for Aesthetic Plastic Surgery.

This is an Open Access article distributed under the terms of the Creative Commons At tribution Non-Commercial License (http://creativecommons.org/licenses/by-nc/4.0/) which permits unrestricted non-commercial use, distribution, and reproduction in any medium, provided the original work is properly cited. $\quad w w w . e-a a p s . o r g$ ing introduced for the reconstruction of breast parenchymal defects after breast cancer surgery. Among them, myocutaneous flaps using the latissimus dorsi (LD) muscle are widely used because of their high vascularity and flexibility that facilitate shape control in breast reconstructive surgery. A drawback of the LD flap is the risk of donor-site morbidity on the back. The most common complication is seroma formation, and the incidence of seroma as a complication has been reported to vary, ranging from $1 \%$ to $56 \%[2,3]$. Despite such a high incidence, only limited research on donor-site seroma formation has been conducted $[4,5]$.

Several mechanisms of seroma formation have been reported. The donor site of an LD myocutaneous flap may have hidden bleeding, and prolonged lymphatic leakage may cause fluid collection. Further, the shearing force generated by the constant movement of a patient can cause a disruption between the skin and the underly- 
ing musculature [6]. Wound dehiscence due to seroma formation not only makes a patient's movement uncomfortable but also increases the infection rate [7].

Fibrin sealant (FS; Baxter Healthcare Corp, Westlake Village, CA, USA) is a material consisting of fibrinogen and thrombin, and is frequently used in efforts to achieve hemostasis and wound adhesion as well as in many medical settings. Since FS has a direct effect on hemostasis, it is effective for bleeding control in internal organs such as the spleen and in the treatment of tissue fluid leakage, such as cerebrospinal fluid leakage, acting as an aseptic adhesive $[8,9]$. FS has been considered to have a clinically meaningful effect on seroma reduction and has received considerable attention from many surgeons.

Several studies reporting that the use of FS in knee surgery was effective in reducing vascular bleeding and routine blood transfusions have been published $[10,11]$. However, thus far, no study on the hemostatic effect of FS on the donor-site morbidity of LD flaps has been reported. In contrast, a number of reports have been published on the use of FS for donor-site seroma prevention in cases of LD flap surgery. Previous reports have stated that a single application of FS was not effective in the reduction of seroma and is thus not recommended [12-14]. One study has also reported that FS was effective in the reduction of seroma [15]. However, no satisfactory conclusion on this issue has been reached as yet. Therefore, in this study, we aimed to investigate the effect of FS on seroma prevention and as a hemostatic agent for the donor site; the study sample consisted of more than 200 patients who underwent treatment at a single clinical institute.

\section{METHODS}

\section{Study design}

This retrospective study based on chart review was conducted among patients who received treatment from January 2011 to August 2015. This study complies with the Declaration of Helsinki and was performed according to ethics committee approval. All the patients received immediate breast reconstruction utilizing an LD myocutaneous island flap. All the patients were provided information about this research prior to surgery. Before the scheduled operation, a blood sample was collected to measure the preoperative hemoglobin $(\mathrm{Hb})$ level. The patients were unaware of the drugs administered in the preoperative period. During the operation, the total weight of the extirpated breast tissue was measured with a consistently used scale. Sentinel lymph node dissection was performed in all the patients. Two 200-mL Jackson-Pratt (J-P) drains (Barovac; Sewoon Medical, Seoul, Korea) were placed in the LD muscle donor site and the donor site with the axilla dissection on the back (Fig. 1). We always used 2 drains in the back. The drains were removed when the drainage amount was less than $15 \mathrm{~mL}$ for 2 consecutive days. The postoperative $\mathrm{Hb}$ level, drain amount, drain re-

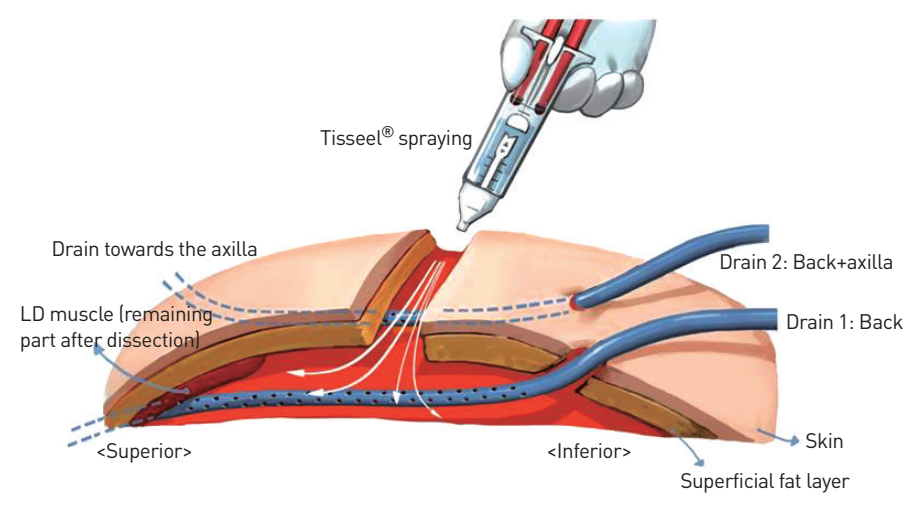

Fig. 1. Insertion of two 200-mL Jackson-Pratt drains in the latissimus dorsi (LD) muscle donor site and the donor site with axillary dissection on the back. Fibrin sealant was sprayed after drain insertion.

moval time, and the seroma incidence rate were investigated by our team. The drain amount was calculated only from the $2 \mathrm{~J}-\mathrm{P}$ tubes in the back area. Seroma was defined as a visual or ultrasonographic observation of fluid collection or fluctuation after drain removal. The exclusion criteria were as follows: a history of preoperative steroid or anticoagulant use; a history of systemic disease, infection, being pregnant or breastfeeding, coagulopathy, or hypersensitivity such as an allergic reaction to bovine protein; and incorrect patient data.

The FS was prepackaged with 2 separate syringes for fibrinogen and thrombin. We sprayed $4 \mathrm{~mL}$ of the aerosolized fibrin on the flap donor site on the back. The same amount of both the components was sprayed at the same speed at the same time. These 2 components mixed to produce a viscous glue that promptly caused coagulation; further, the presence of thrombin facilitated the conversion of fibrinogen into fibrin. It should be noted that the donor site was compressed manually for 5 minutes to help the absorption of FS.

The results of a comparison of the effects of FS were divided into 2 phases: the early phase was defined as within 1 week of the operation, and the late phase was defined as 1 week after surgery.

\section{Statistical analysis}

We first analyzed the normal distribution of the collected data and compared the mean values between the 2 groups. Mean differences between the groups were analyzed by the independent $t$-test. In the case of a nominal scale, such as seroma incidence, we performed the chi-square test to make comparisons between the groups. SPSS version 22 (IBM Corp., Armonk, NY, USA) was used for the data analysis. All P-values of less than 0.05 were considered to indicate statistical significance.

\section{RESULTS}

Data from 250 women were collected for this trial. Three patients 
Table 1. Patient demographics

\begin{tabular}{lc}
\hline & Mean $(\mathrm{n}=234)$ \\
\hline Age (year) & 46.78 \\
Body mass index $\left(\mathrm{kg} / \mathrm{m}^{2}\right)$ & 23.53 \\
Extirpated breast weight (g) & 182.25 \\
Hemovac removal day (day) & 18.55 \\
Operation time (minute) & 327.77 \\
Seroma (\%) & 29.49 \\
\hline
\end{tabular}

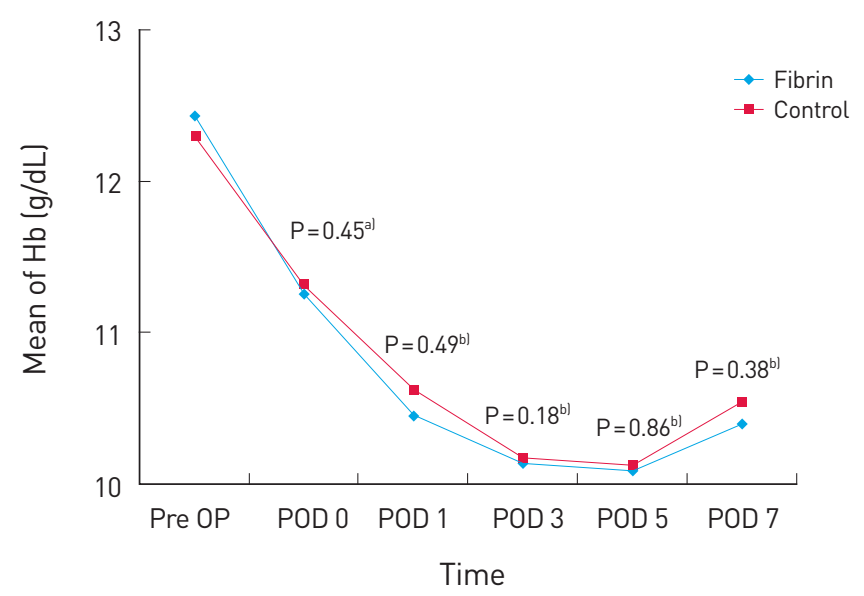

Fig. 2. Changes in the hemoglobin $(\mathrm{Hb})$ level at different postoperative days (PODs). The level decreased gradually up to POD 5, and then recovered. ${ }^{a} \mathrm{As}$ a value indicating the difference of $\mathrm{Hb}$, defined as the value obtained by subtracting $\mathrm{Hb}$ of POD 0 day from $\mathrm{Hb}$ of preoperative state. ${ }^{\text {bl}} \mathrm{As}$ in the case of ${ }^{\text {al }}$, it is obtained by subtracting the backward value from the previous value. OP, operation.

were administered a $2 \mathrm{~mL}$ dose of FS, and 13 patients whose drain removal time was not accurate were excluded from this study. The patient demographic data are summarized in Table 1. The sample for the final analysis consisted of 234 patients. The mean patient age was 46.78 years, and the mean value of the body mass index was $23.53 \mathrm{~kg} / \mathrm{m}^{2}$. Of the sample, 164 patients were sprayed with aerosolized FS, and 70 patients underwent surgery without FS (control group). We also compared the drain removal time, seroma rate, postoperative $\mathrm{Hb}$ level change, and the drain amount between the 2 groups.

$\mathrm{Hb}$ levels in patients after surgery were measured on postoperative day (POD) 0, POD 1, POD 3, POD 5, and POD 7. Upon analyzing the changes in the $\mathrm{Hb}$ level, we found that both the FS group and the control group showed the same pattern of a gradual decrease up to POD 5, followed by recovery. The $\mathrm{Hb}$ level was not statistically significantly different between the 2 groups from POD 0 to POD 7. However, on POD 0 and POD 1, the FS group showed a greater decrease in the $\mathrm{Hb}$ level (Fig. 2).

The mean drain removal time was 18.55 days. Upon comparing the daily drain amount between the 2 groups from POD 0 to POD

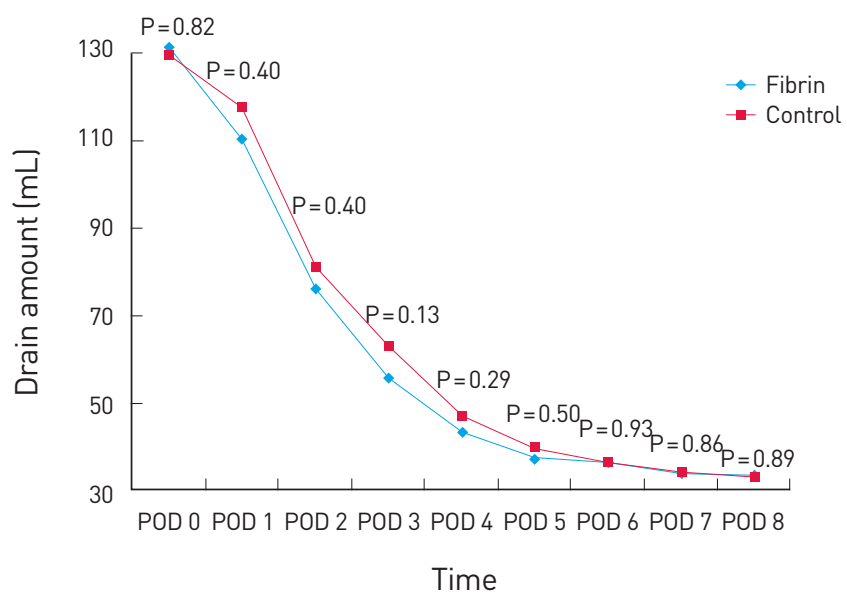

Fig. 3. Changes in the drain amount on different postoperative days (PODs). Except for POD 0, a higher drain amount was observed in the control group.

8 , we found that the mean value of the drain amount was not lower in the FS group (Fig. 3). The drain amount was not statistically significantly different between the 2 groups, but was higher in the FS group on POD 0 (Fig. 3). The mean drain removal time was 20.06 days in the FS group and 17.51 days in the control group, but this difference was not statistically significant $(\mathrm{P}=0.11)$. The overall seroma incidence rate was $29.49 \%$. The seroma incidence rate was higher in the FS group than in the control group, but not to a statistically significant extent $(\mathrm{P}=0.70)$.

\section{DISCUSSION}

Thus far, various methods for seroma prevention have been introduced, such as intraoperative electrocautery, endoscopic harvest, compression garment, repetitive aspiration, sclerotherapy, steroid injections, and prolonged hemovac drainage; however, none of these methods have shown a marked effect [16]. Therefore, many surgeons are interested in methods for seroma reduction at the LD donor site for breast reconstructive surgery.

Upon comparing the decrease in the patients' $\mathrm{Hb}$ levels on different PODs between the 2 groups, we found no statistically significant difference between the FS group and the control group. This result indicates that FS was not effective in reducing postoperative bleeding. As $\mathrm{Hb}$ is known to start to decrease 24 to 48 hours after bleeding, its level does not represent immediate postoperative bleeding [17]. Further, changes in the Hb level 24 to 48 hours after bleeding have been widely used as universal indicators of actual bleeding, because the loss of the extracellular fluid caused by dehydration or volume replacement and the changes in the plasma amount [10]. Although not all the factors that can cause an acute change in the $\mathrm{Hb}$ level have been investigated, the fasting period of the patients and the types and amounts of injection fluid administered to 
the patients were almost the same; thus, these factors were thought to not have an impact on the results. Therefore, we used changes in $\mathrm{Hb}$ levels after 24 to 48 hours as a tool to determine the effect of hemostasis.

Several studies have reported that the use of FS in orthopedic knee surgery was effective in reducing vascular bleeding and the need for routine blood transfusion $[10,11]$. FS reduced the complications related to blood transfusions and decreased the costs related to the transfusions and acute postoperative bleeding control. However, certain differences between orthopedic knee surgery and breast reconstruction surgery make FS ineffective at the LD donor site. In the case of orthopedic knee surgery, massive bleeding due to the tearing of a large blood vessel is possible in small operative fields. LD flap harvest involves several perforators and a relatively large operative field. These perforators do not cause massive bleeding. Therefore, muscle bleeding takes place instead of massive bleeding such as diffuse oozing, and it is therefore difficult for fibrin to successfully cause or accelerate hemostasis. Moreover, the shearing force caused by the constant movement of the patients interferes with the action of the fibrin. Therefore, we conclude that the hemostatic effect of fibrin does not prevent LD donor-site morbidity.

A comparison of the drain amount revealed no statistically significant difference between the 2 groups. This indicates that FS cannot decrease secretions from a microvessel or lymphatic vessel in the early phase. The drain removal time can reflect the amount of drainage in the late phase. In the present study, we did not observe a statistically significant difference in the drain removal time, so we inferred that FS did not reduce the drainage amount, as the adhesive sealant blocked the lymphatic channel in both the early and the late phase. This might be attributed to the re-opening of the lymphatic system after surgery, irrespective of the use of FS [12,18].

Quilting sutures can be a procedure that facilitates seroma prevention in LD donor sites. Many researchers, through systemic reviews and meta-analyses, have shown the effectiveness of a combination of quilting sutures and FS [19-23]. In these studies, a combination of quilting sutures and FS significantly reduced the seroma rate, total drainage amount, drain removal time, and frequency of seroma aspiration. The fixation effects of a quilting suture with the underlying musculature may maximize the fibrin adhesion. Further, a quilting suture can successfully obliterate any dead space with tissue fixation and minimize the shearing force of the skin flap and the underlying structures [19-21]. Tissue adhesion with a quilting suture and the immobilization of the patient's shoulder can considerably minimize any dead space and the shearing force [2022]. Quilting sutures can also reduce the spontaneous movement between the skin flaps and the underlying muscle, thus decreasing inflammation. The redistributed tension of the back donor site decreases the tension on the incision site and may prevent the spread of the resultant scar [20].

In the present study, the ineffectiveness of FS with respect to se- roma prevention could be attributed to several reasons. First, we think that the area of the back donor site is too large to be covered with only $4 \mathrm{~mL}$ of FS; further, it is difficult to predict which part will have reactive fluids or bleeding when applying FS. Second, since reactive fluid formation occurs frequently in the back area and leads to a drainage amount of tens of milliliters a day, it is difficult for FS to have a distinct adhesive effect [18]. Third, as the constant movement of the patient's shoulder and flank area generates a shearing effect between the skin and the underlying musculature, it is difficult to have the same effect as that of an FS application to a small area such as an internal organ or a vessel that has minimal movement. Fourth, we used $4 \mathrm{~mL}$ of FS empirically; the recommended dose of FS for the back area has not been established as yet. Therefore, the use of FS alone had no effect on seroma prevention, and an additional procedure may need to be performed to complement the effect of FS.

Considerable research has been conducted to determine whether FS affects seroma prevention. However, there is significant methodological and clinical diversity in these reports $[7,14,15,24]$. In some reports, both immediate and delayed breast reconstructions were included [15], and in certain reports, if the drains were retained even after 10 days of surgery, the patient was considered to have developed seroma [14]. Further, on the basis of a review of 19 large randomized controlled trials involving seroma, Sajid et al. [24] claimed that fibrin glue does not affect postoperative seroma. They pointed out that the trials were conducted in an uncontrolled environment, and thus, there may be significant bias. Therefore, in the future, a controlled trial is needed to minimize such bias.

Our study has several advantages compared with the previous studies on LD donor-site seroma. First, we considered immediate breast reconstructions performed by a single surgeon using the same surgical method. The surgery was performed in the same operation room and with similar timing. The same medical team also performed wound care and nursing care consistently. During data collection, multiple researchers collected the data to prevent selection bias. We were thereby able to minimize the technical and methodological errors discussed in previous reports [24]. Second, in this study, we considered a large patient pool of more than 200 patients who underwent $L D$ reconstruction. In particular, of the studies on the effects of FS on postoperative complications after breast reconstruction with an LD flap, this study had the largest patient pool. Therefore, the results of this study can be considered more reliable. Third, this is the first study to investigate the hemostatic effect of FS on LD donor sites. Since FS has no hemostatic effect, meticulous bleeding control is important in the intraoperative field and the empirical use of FS should be avoided. However, this study has several limitations. First, this study is a retrospective one; the causality of spraying FS should be investigated in a prospective, randomized, double-blinded study. Second, the number of patients in the FS group was more than twice the number in the control group. 
Third, a relatively small dose of FS ( $4 \mathrm{~mL})$ was used in this study. This dosage reflected empirical use of FS, and a larger dose of FS may be required in the large back area; therefore, studies on the appropriate dosage of FS need to be conducted in the future.

\section{CONCLUSION}

FS does not have seroma prevention, postoperative hemostatic, or adhesive sealing effects. It works as a part of a foreign body reaction for fluid formation several weeks after surgery. We conclude that the empirical use of FS alone did not result in seroma reduction and hemostasis. We believe that this result will help to reduce the futile empirical use of FS in the future. Several researchers have reported that shoulder immobilization and quilting sutures had a positive effect on seroma prevention. In the future, the effects of a combination of quilting sutures and FS should be studied. Future research should employ a prospective, randomized, double-blinded study design, in order to address some limitations of this study.

\section{PATIENT CONSENT}

Patients provided written consent for the use of their images.

\section{REFERENCES}

1. Jung KW, Won YJ, Kong HJ, et al. Cancer statistics in Korea: incidence, mortality, survival, and prevalence in 2012. Cancer Res Treat 2015;47: 127-41.

2. Davis JP, Nield DV, Garth RJ, et al. The latissimus dorsi flap in head and neck reconstructive surgery: a review of 121 procedures. Clin Otolaryngol Allied Sci 1992;17:487-90.

3. Salmi A, Tukiainen E, Harma M, et al. A prospective study of changes in muscle dimensions following free-muscle transfer measured by ultrasound and CT scanning. Plast Reconstr Surg 1996;97:1443-50.

4. Jeon BJ, Lee TS, Lim SY, et al. Risk factors for donor-site seroma formation after immediate breast reconstruction with the extended latissimus dorsi flap: a statistical analysis of 120 consecutive cases. Ann Plast Surg 2012;69:145-7.

5. Tomita K, Yano K, Masuoka T, et al. Postoperative seroma formation in breast reconstruction with latissimus dorsi flaps: a retrospective study of 174 consecutive cases. Ann Plast Surg 2007;59:149-51.

6. Scheflan M, Kalisman M. Complications of breast reconstruction. Clin Plast Surg 1984;11:343-50.

7. Sajid MS, Betal D, Akhter N, et al. Prevention of postoperative seroma-related morbidity by quilting of latissimus dorsi flap donor site: a systematic review. Clin Breast Cancer 2011;11:357-63.

8. Canby-Hagino ED, Morey AF, Jatoi I, et al. Fibrin sealant treatment of splenic injury during open and laparoscopic left radical nephrectomy.
J Urol 2000;164:2004-5.

9. Siedentop KH, O’Grady K, Park JJ, et al. Fibrin sealant for treatment of cerebrospinal fluid leaks. Am J Otol 1999;20:777-80.

10. Sabatini L, Trecci A, Imarisio D, et al. Fibrin tissue adhesive reduces postoperative blood loss in total knee arthroplasty. J Orthop Traumatol 2012;13:145-51.

11. Yang TQ, Geng XL, Ding MC, et al. The efficacy of fibrin sealant in knee surgery: A meta-analysis. Orthop Traumatol Surg Res 2015;101: $331-9$.

12. Vaxman F, Kolbe A, Stricher F, et al. Does fibrin glue improve drainage after axillary lymph node dissection? Prospective and randomized study in humans. Eur Surg Res 1995;27:346-52.

13. Llewellyn-Bennett R, Greenwood R, Benson JR, et al. Randomized clinical trial on the effect of fibrin sealant on latissimus dorsi donorsite seroma formation after breast reconstruction. Br J Surg 2012;99: $1381-8$.

14. Miri Bonjar MR, Maghsoudi H, Samnia R, et al. Efficacy of fibrin glue on seroma formation after breast surgery. Int J Breast Cancer 2012;2012: 643132

15. Weinrach JC, Cronin ED, Smith BK, et al. Preventing seroma in the latissimus dorsi flap donor site with fibrin sealant. Ann Plast Surg 2004; 53:12-6.

16. Dancey AL, Cheema M, Thomas SS. A prospective randomized trial of the efficacy of marginal quilting sutures and fibrin sealant in reducing the incidence of seromas in the extended latissimus dorsi donor site. Plast Reconstr Surg 2010;125:1309-17.

17. Turgeon ML. Clinical hematology: theory and procedures. 4th ed. New York, NY: Lippincott Williams \& Wilkins; 2005.

18. Cha HG, Kang SG, Shin HS, et al. Does fibrin sealant reduce seroma after immediate breast reconstruction utilizing a latissimus dorsi myocutaneous flap? Arch Plast Surg 2012;39:504-8.

19. Lee KT, Mun GH. Fibrin sealants and quilting suture for prevention of seroma formation following latissimus dorsi muscle harvest: a systematic review and meta-analysis. Aesthetic Plast Surg 2015;39:399-409.

20. Bailey SH, Oni G, Guevara R, et al. Latissimus dorsi donor-site morbidity: the combination of quilting and fibrin sealant reduce length of drain placement and seroma rate. Ann Plast Surg 2012;68:555-8.

21. Shin IS, Lee DW, Lew DH. Efficacy of quilting sutures and fibrin sealant together for prevention of seroma in extended latissimus dorsi flap donor sites. Arch Plast Surg 2012;39:509-13.

22. Daltrey I, Thomson H, Hussien M, et al. Randomized clinical trial of the effect of quilting latissimus dorsi flap donor site on seroma formation. Br J Surg 2006;93:825-30.

23. Ali SN, Gill P, Oikonomou D, et al. The combination of fibrin glue and quilting reduces drainage in the extended latissimus dorsi flap donor site. Plast Reconstr Surg 2010;125:1615-9.

24. Sajid MS, Hutson KH, Rapisarda IF, et al. Fibrin glue instillation under skin flaps to prevent seroma-related morbidity following breast and axillary surgery. Cochrane Database Syst Rev 2013;5:CD009557. 